Meta

Journal des traducteurs

Translators' Journal

\title{
L’ambiguïté : un défi traductologique
}

\section{Ronald Landheer}

Volume 34, numéro 1, mars 1989

Humour et traduction

Humour and Translation

URI : https://id.erudit.org/iderudit/003395ar

DOI : https://doi.org/10.7202/003395ar

Aller au sommaire du numéro

Éditeur(s)

Les Presses de l'Université de Montréal

ISSN

0026-0452 (imprimé)

1492-1421 (numérique)

Découvrir la revue

Citer cet article

Landheer, R. (1989). L’ambiguité : un défi traductologique. Meta, 34(1), 33-43.

https://doi.org/10.7202/003395ar d'utilisation que vous pouvez consulter en ligne.

https://apropos.erudit.org/fr/usagers/politique-dutilisation/ 


\section{L'AMBIGUIITÉ : UN DÉFI TRADUCTOLOGIQUE}

RONALD LANDHEER Université de Leiden, NL

«... le péril majeur est le dogme a priori de l'intraduisibilité». Mounin 1963: 275 .

\section{INTRODUCTION}

La plupart des traductologues ont beau rejeter actuellement la fameuse «objection préjudicielle» (cf. Ladmiral 1979:85 sq.), qui pose que la traduction est impossible et que, au fond, tout est «intraduisible», cela n'empêche pas que la notion d'intraduisibilité réapparaît couramment, aussitôt qu'il s'agit de la traduction d'ambiguités voulues, de jeux de mots ou d'autres formes de l'humour verbalisé.

Le passage suivant - bien qu'il ne se rapporte pas à la traduction du discours humoristique proprement dit $^{1}$, mais à celle des figures de mots du type

(1) Traduttore, traditore est éloquent à cet égard :

«Partons de la maxime italienne: Traduttore, traditore; elle est proprement intraduisible. Si je dis : traducteur, traître, je perds la répétition de syllabes (paronomase) qui fait sa force. La première caractéristique des figures de mots est qu'elles sont intraduisibles, à moins qu'on ne trouve par hasard leur équivalent dans une autre langue.» (Reboul 1984 : 37)

Malheureusement les traducteurs ne sont que trop portés à abonder dans le sens de Reboul, et à invoquer le principe de la non-traductibilité dans les cas où ils n'ont pas eu ou pris le temps de chercher un équivalent adéquat dans la langue cible.

À notre avis, la thèse de Reboul au sujet de ce qu'il appelle les figures de mots ${ }^{2}$ est formulée trop à la légère et repose sur un a priori. Pour reprendre son exemple, en effet : «traducteur, traître» comme traduction (ou plutôt comme transcodage dans la terminologie de Seleskovitch et Lederer 1984) de (1) n'est certainement pas la meilleure des solutions, pour la raison évoquée par l'auteur. Mais il n'est pas trop difficile d'y substituer une autre traduction, entièrement satisfaisante, qui restitue à la fois l'essence du message ${ }^{3}$ et son expression rhétorique, a savoir :

(1a) Traduction, trahison.

Il ne nous a pas fallu plus de quelques minutes pour la trouver (aidé ou non par le «hasard», on ne le saura jamais...), avant de rencontrer, quelque temps après, cette même traduction dans une étude de Michel Ballard 1987.

«Les figures les plus expressives», continue Reboul, toujours à propos des figures de mots, «sont celles qui concernent le rythme de la phrase». «Sans lui, dit Cicéron, le sens reste le même, non l'effet», et Reboul cite comme exemple le slogan suivant, comportant deux fois quatre syllabes:

(2) Boire ou conduire, il faut choisir (ibid. : 37-38).

Or, cet exemple n'est pas plus intraduisible que l'exemple précédent, du moins en néerlandais ${ }^{4}$, où un parfait équivalent serait : 
(2a) Drinken of rijden, 't is één van beide

(= «boire ou conduire, de deux choses l'une»),

équivalent qui garde à la fois la rime (rijden - dont le $\mathrm{n}$ final ne se prononce pas - et beide), la symétrie rythmique $(5+5)$ et la valeur sémantique et pragmatique du message.

Bien entendu, nous n'aurons pas l'audace de prendre le contrepied de Reboul et de tant d'autres auteurs qui ont tendance à insister sur le caractère essentiellement intraduisible de ce type de discours 5 . En effet, il serait peu réaliste de soutenir que le discours ambigu, équivoque ou humoristique, puisse toujours être traduit sans perte aucune. Justement il importe de se défaire de telles idées «généralisantes» et de se baser autant que possible sur les faits, c'est-à-dire d'une part, l'existence de traductions très réussies, et d'autre part, des «ratés» évidents. À partir de ces faits, on peut essayer d'entrer dans le vif de la problématique.

Étant donné que l'ambiguitté est en général considérée - et à juste titre - comme un des grands obstacles de la traduction, automatique aussi bien qu'humaine, nous voudrions dans ce qui suit passer en revue les différents problèmes auxquels le traducteur peut être confronté, lorsqu'il se trouve devant un énoncé ambigu. Nous nous occuperons surtout de l'ambiguité voulue, intentionnelle, parce que c'est elle qui est à la base, le plus souvent, du discours humoristique - le thème central de ce numéro de META - et que c'est elle aussi qui pose au traducteur les plus grands problèmes. Nous tâcherons de formuler des hypothèses sur la stratégie qui nous paraît recommandable pour le traducteur devant la diversité de ses occurrences et sur les principaux facteurs déterminant sa traduisibilité.

\section{AMBIGUÏTÉ ET TRADUCTION}

Pour ce qui regarde l'ambiguïté, il y a notamment trois aspects qui méritent quelques remarques préalables: le côté énoncé, le côté émetteur et le côté récepteur (ou traducteur).

\section{a) Le côté énoncé}

Un énoncé peut être ambigu

I. dans la langue parlée et dans la langue écrite à la fois :

(3) Le secrétaire est dans le bureau

(4) Georges aime Marie autant que Jean',

II. dans le code parlé seulement:

(5) [lalivretuver] (le livre est tout vert / le livre est ouvert),

III. dans le code écrit seulement:

(6) Vous connaissez tous les effets de cette maladie ([tu] / [tus]).

Il va sans dire que le traducteur aura surtout ${ }^{7}$ affaire aux types I et III, l'interprète aux types I et II.

Jusqu'ici les problèmes sont de nature purement langagière.

\section{b) Le côté émetteur}

Du point de vue de l'émetteur, il convient de distinguer entre

1. l'ambiguité intentionnelle et

2. l'ambiguïté non intentionnelle.

Une ambiguité intentionnelle est une ambigüté préméditée, manifestement voulue par l'émetteur. «Manifestement» en effet, car normalement il y a dans le contexte une marque d'intentionnalité ${ }^{8}$, c'est-à-dire des signaux ou indices qui doivent nous rendre conscients de telles ambiguïtés. Certains textes ou sortes de textes sont d'ailleurs plus productifs à cet égard que certains autres : la poésie, la publicité, le Canard enchaîné, etc. 
Une ambiguïté non intentionnelle est une ambiguiité quelque part dans le message, dont il est évident ou très probable que l'émetteur ne se rend pas compte; elle est donc involontaire et ne présente aucune valeur fonctionnelle dans le discours en question. Dans la communication normale, monolinguale, il y a alors deux possibilités : ou bien, on identifie ce que l'émetteur a voulu dire, grâce à la situation ou à une sorte de calcul de probabilités, comme dans l'exemple suivant, souvent cité :

(7) À vendre bicyclette de dame ayant peu roulé

(Le Goffic 1981 : 246),

ou bien, on n'identifie pas le sens de l'énoncé, qui reste cryptique pour le destinataire. Dans ce dernier cas, la communication est perturbée. Il est clair que ces deux possibilités peuvent se présenter aussi dans la communication interlinguale, celle du traducteur. Nous y reviendrons dans le paragraphe suivant.

\section{c) le côté récepteur-traducteur}

$\mathrm{Au}$ récepteur se pose tout d'abord le problème de l'identification. Il doit essayer de bien distinguer entre ambiguiités intentionnelles et ambiguïtés non intentionnelles. Quelle doit être la stratégie du récepteur-traducteur dans ces deux cas? Dans le paragraphe trois, nous nous proposons de faire l'inventaire des modalités qui peuvent se produire.

\section{INTENTIONNALITÉ ET STRATÉGIE DU TRADUCTEUR}

Le traducteur confronté à un énoncé ambigu, doit traverser deux phases cruciales: $1^{\circ}$ une phase de compréhension ou d'interprétation, non seulement de l'énoncé, mais encore de l'intentionnalité de $1^{\prime}$ 'énonciateur, et $2^{\circ}$ une phase de restitution, soit «correctrice», soit «recréatrice». C'est que sa stratégie doit être tout à fait différente selon qu'il a à faire à une ambiguïté non intentionnelle ou à une ambiguïté intentionnelle.

\section{1) L'ambiguitté non intentionnelle: la traduction «correctrice»}

Le traducteur aura soin de désambiguïser, dans la mesure du possible, les ambiguïtés non intentionnelles. C'est que, normalement parlant, elles ne doivent jamais être traduites en tant que telles. Du point de vue linguistique, il n'est pas difficile de désambiguïser des phrases-énoncés (3)-(6). S'il s'agit là manifestement d'ambiguïtés involontaires, qui - intégrées à un contexte quelconque — n'ont aucune fonction communicative spéciale, il serait peu recommandable de vouloir pousser la fidélité à l'original assez loin pour maintenir de telles ambiguités locales dans le texte cible.

D'ailleurs, il convient de faire ici une distinction importante concernant la transparence relative du contexte. Si les phrases citées se rencontrent dans un contexte (relativement) clair, transparent, si en d'autres termes il s'agit seulement de phrases ambiguës, et non d'énoncés ambigus, alors le traducteur peut et doit désambiguïser la phrase en question en choisissant le sens approprié dans le texte d'arrivée. Si, par contre, le contexte est (assez) obscur, si bien que l'énoncé est (presque) aussi ambigu que la phrase, la «faute» en est à l'émetteur: c'est un cas de communication ratée (cf. le paragraphe précédent). Le traducteur pourra choisir le sens qui lui paraît le plus probable en l'occurrence, ou éventuellement - si cela est linguistiquement possible - il pourra laisser subsister l'ambiguïté en question dans le texte cible (et informer le lecteur concernant l'opacité du texte de départ, p. ex. au moyen d'une note explicative). Ainsi l'exemple (4) peut être restitué littéralement en anglais :

(4a) Georges loves Mary as much as John, mais non pas en néerlandais, où une désambiguïsation s'impose. Le traducteur doit choisir entre :

(4b 1) Sjors houdt evenveel van Marie als Jan et 
(4b 2) Sjors houdt evenveel van Marie als van Jan.

Même si le contexte ne lui permet guère d'entrevoir lequel des deux sens serait le plus probable, il est obligé de trancher!

Dans la littérature, de tels cas d'ambiguïté non intentionnelle, et indécidables (ou presque) pour le traducteur, sont rares, mais ils se rencontrent, comme l'a montré Michel Ballard (op. cit.) à propos de

(8) a large check suit (dans un roman de H.G. Wells, traduit par U. Ballard, 1984),

dont il n'est pas clair si large se rapporte à check ou à suit.

\section{2) L'ambigüité intentionnelle : la traduction «recréatrice»}

La stratégie du traducteur à l'égard des ambiguïtés intentionnelles doit être tout autre. C'est qu'il ne doit jamais tenter de désambiguïser ces énoncés, mais, au contraire, faire tout son possible pour restituer l'ambiguïté du texte-source en la traduisant par une ambiguitté analogue dans le texte-cible. À noter que nous disons «analogue» et non pas «identique»: nous voyons dans la traduction une paraphrase interlinguale, conçue comme un équivalent communicatif et non comme un «énoncé synonyme» de l'énoncé de départ 9 .

Si la traduction paraît être vraiment impossible, le traducteur aura soin, ou bien de la compenser d'une manière ou d'une autre, par exemple en évoquant un effet analogue dans le contexte immédiat, ou bien de l'expliciter au moyen d'une note critique (ce qui est un pis-aller!).

En revanche, l'omission, sans plus, d'une ambiguïté intentionnelle amène toujours une perte sensible dans la traduction, comme le montrent les deux exemples suivants:

(9) Mais, à l'instar des astronautes, qui, à partir d'une certaine distance, échappent aux obligations de la pesanteur, je ne me sens plus soumis - dès que je suis projeté sur le Continent - aux lois de la gravité britannique. (Daninos, les Cahiers du Major Thompson, 1973, pp. 9-10.)

Le mot gravité a été rendu dans la traduction néerlandaise par ernst (= «sérieux», «componction»), si bien que l'autre sens de gravité («gravitation»), actualisé ici par la cooccurrence des mots pesanteur et astronautes, se perd complètement. Pourtant une traduction plus adéquate aurait été possible, à savoir zwaarwichtigheid, qui veut dire aussi «componction», mais qui a un signifiant assez transparent pour renvoyer aussi le lecteur au sens de «pesanteur», «gravitation».

(10) Vous réfléchissez? moi aussi, des fois. Mais dans un miroir. (Ionesco, «Jacques ou la soumission», dans Théâtre I, p. 119.)

Ici le traducteur néerlandais ne donne qu'un seul sens de réfléchir, à savoir l'équivalent hollandais de «penser». Ainsi, la seconde isotopie, celle de la réflexion lumineuse, actualisée par le mot miroir disparait. Pourtant, ici encore, une alternative bien plus satisfaisante se présente, sous la forme suivante:

(10a) Staat $u$ wel eens ergens bij stil? Ik ook soms. Bij de spiegel. (stilstaan a les deux sens de «s'arrêter devant» et «réfléchir à»).

Ces sortes de solutions ne sont d'ailleurs jamais automatiques, mais dépendent très fortement du contexte. Ainsi, dans l'exemple suivant, une ambiguïté comparable avec le mot réfléchir ne saurait être restituée de la même façon:

(11) Les miroirs feraient bien de réfléchir avant de nous renvoyer notre image. (J. Cocteau)

Ici une traduction convenable serait :

(11a) Spiegels zouden zich wel twee keer moeten bedenken...,

où la notion du «double» est évoquée par twee keer (= [réfléchir] «deux fois»). 
Il est clair que, en escamotant ces ambiguìtés conscientes, sous prétexte (formulé ou non) qu'elles sont intraduisibles, le «traducteur trahit» (en effet!) sa haute mission.

Résumons-nous. L'ambiguiité non intentionnelle, qu'elle soit effective ou non, ne doit pas être préservée en tant que telle dans le texte d'arrivée. Le traducteur fera bien de choisir le sens le plus probable, ou, en cas de doute, d'expliciter ses doutes de manière ou d'autre.

L'ambiguïté intentionnelle - qu'elle ait une fonction communicative, argumentaire, esthétique ou ludique - mérite une attention spéciale de la part du traducteur, comme de la part du traductologue. Elle doit être traduite de façon à ce qu'elle produise sur les récepteurs du texte-cible un effet analogue à celui qu'elle provoque sur les récepteurs du texte original. Ce serait un truisme, si les traducteurs n'étaient pas si souvent convaincus de la quasi-impossibilité de traduire adéquatement ce type de discours. Il s'agit là d'un préjugé tenace, qui nuit considérablement à la qualité du texte traduit.

Dans le paragraphe suivant, nous nous proposons d'évaluer les particularités des différents types d'ambiguïtés intentionnelles, dont l'isotopie (au moins) double provoque la plupart du temps un effet humoristique.

\section{TYPES D'AMBIGUIITÉS INTENTIONNELLES}

Ailleurs (Landheer 1984:5) nous avons adopté la définition suivante d'un jeu de mots : «Un jeu de mots est un énoncé qui contient un élément (ou plusieurs éléments de forme identique ou quasi identique) dont la plurivalence a été consciemment exploitée par l'émetteur».

Comme le jeu de mots contient toujours une ambiguité consciente ${ }^{10}$ et qu'il produit presque toujours un effet humoristique, c'est notamment sur ce type de discours que nous voudrions fixer notre attention maintenant. Apportons tout d'abord quelques précisions à propos de la définition citée.

Lorsqu'il est question d'un élément plurivalent, il s'agit d'un terme polysémique: (9), (10) et (11) en étaient des exemples; nous considérons gravité et réfléchir comme des lexèmes polysémiques, dont les différentes acceptions sont reliées ${ }^{11}$.

Lorsqu'il est question de plusieurs éléments de forme identique, il s'agit de termes homonymiques ou homophoniques (ou éventuellement homographiques-non homophoniques comme dans l'exemple $\left.(6)^{12}\right)$.

\section{Homonimique :}

(12) Mourir: se mettre aux vers (Queneau, les Euvres complètes de Sally Mara, $335^{13}$.

\section{Homophonique :}

(13) Entre deux mots il faut choisir le moindre (mot attribué à Valéry) 14 .

Lorsqu'il est question de plusieurs éléments de forme quasi identique, il s'agit de termes paronymiques:

(14) Voyage au bout de l'ennui (cf. Céline : la Nuit) ${ }^{15}$

$\bar{A}$ propos des exemples que nous venons de citer: (9)-(14), il convient de faire encore deux distinctions importantes :

A) Les cas cités appartiennent tous à la catégorie des ambiguintés in absentia (Hausmann 1974 parle dans ce cas de jeux verticaux), c'est-a-dire que la plurivalence des éléments y est exploitée implicitement (même si elle est actualisée par des éléments cooc- 
currents). Mais la plurivalence peut être exploitée aussi d'une manière explicite, dans des ambiguités in praesentia (ou horizontales). Voici des exemples:

\section{Polysémie :}

(15) Colin montait, le nez sur les talons des deux filles. De jolis talons renforcés, en nylon clair.

(Vian, l'Écume des jours, 41) 16

(16) Tu pleureras l'heure où tu pleures. (Apollinaire, «À la santé», Alcools.) $)^{17}$

\section{Homonymie :}

(17) Une orange sur la table / Ta robe sur le tapis / Et toi dans mon lit / Doux présent du présent / Fraîcheur de la nuit / Chaleur de ma vie (Prévert, «Alicante», Paroles $)^{18}$

(18) Les grands mots entraînent toujours de grands maux. (A. France) $)^{19}$

\section{Paronymie :}

(1) Traduttore, traditore (cf. supra),

(19) À mi-chemin de la cage au cachot la langue française a cageot... (Ponge, «Le cageot», le Parti pris des choses) ${ }^{20}$

Ce type de jeux de mots est moins ambigu que celui des jeux in absentia. C'est que, en général, il n'est pas trop difficile de découvrir (dans p. ex. (16) et (17) laquelle des deux valeurs différentes est représentée par la première occurrence et laquelle par la seconde ${ }^{21}$. Mais, comme nous l'avons exposé ailleurs (Landheer, ibid.: 7), l'ambiguïté d'un énoncé comme (17) Doux présent du présent «ne découle pas en premier lieu de la plus ou moins grande incertitude du récepteur concernant l'interprétation du segment répété, mais elle provient avant tout (...) de leur caractère métalinguistique (...). On a beau «paraphraser» Doux présent du présent par «doux cadeau du présent», ces deux syntagmes sont loin d'être sémantiquement identiques. À un sens banal s'associe un deuxième sens, une deuxième lecture, où une information métalinguistique (coulée dans une forme prégnante) constitue l'essentiel. C'est pourquoi une traduction désambiguïsante, qui donnerait seulement l'équivalent de «doux cadeau du présent», serait absolument déficiente!

B) Une seconde distinction à opérer concerne la façon soit directe (ou cumulative), soit indirecte (ou allusive) dont l'ambiguiité est réalisée. Dans le cas d'une ambiguïté cumulative, l'émetteur nous impose deux interprétations à la fois. L'énoncé (10) serait incompréhensible ou de toute façon incohérent, si on ne tenait pas compte de l'ambivalence de réfléchir. Par contre, (13) ou (14) pourraient éventuellement être interprétés et compris en soi. Seulement cette interprétation-là serait lacunaire, parce que leur sens profond réside dans une allusion à d'autres textes, dont ils constituent des déformations voulues. Nous parlons dans ce cas d'une ambiguïté allusive; elle est une réalisation du phénomène de l'intertextualité. Le signal de cette déformation est donné dans leur anomalie relative, mais il fonctionne seulement si le récepteur dispose d'un bagage culturel suffisant. Il y a aussi des cas où l'ambiguïté allusive n'est pas d'ordre intertextuel, mais d'ordre métalinguistique (comme on l'a vu) : (1), (17) et (18) par exemple. Dans tous les cas, une traduction qui négligerait cette ambiguiité allusive, perdrait un aspect important de la valeur totale du message.

Il existe encore une troisième possibilité : c'est qu'une ambiguîté peut encore être sélective; c'est le cas si un énoncé nous impose un choix entre deux interprétations qui 
s'accordent mal ou qui s'excluent. Le plus souvent ces sortes d'ambiguïtés ne sont pas intentionnelles, mais parfois elles le sont bel et bien, comme le montre l'exemple suivant:

(20) It so happens that if there is any institution which is not susceptible to any improvement whatsoever, it is the House of Peers. (Kelly 1971 : 5-6)

Cette «House of Peers» est donc ou bien «parfaite» ou bien «incorrigible». Ces sortes d'assertions ont une ambivalence argumentative qui les rend inattaquables: à quelqu'un qui se sentirait scandalisé, on peut toujours répliquer qu'il a mal interprété...

Ayant passé en revue ces différents types d'ambiguités (tous des jeux de mots, sauf (20), la question qui reste est celle de savoir quels seraient bien les facteurs qui déterminent la réussite (relative) ou la non-réussite (relative) de leur traduction.

\section{LA TRADUCTION DE L'AMBIGUÏTÉ-JEU DE MOTS}

Les ambiguités-jeux de mots touchent aux structures de surface des langues et on sait à quel point les langues sont différentes sous ce rapport. Il y a des langues dont l'ordre des mots est relativement libre, d'autres où il est soumis à des règles très strictes; des langues ayant une dérivation et une composition riches, d'autres dont celles-ci sont plutôt pauvres. Certaines langues ont une polysémie abondante, d'autres encore présentent une profusion d'homonymes. Il y a des langues où l'enchaînement des mots et des morphèmes dans la phrase est si fort que leurs frontières sont à peine perceptibles ; d'autres sont bien plus transparentes à cet égard, etc. Or, le fonctionnement des jeux de mots dépend justement de tous ces facteurs de surface. Pour prendre un exemple concret, une langue qui, comme l'anglais, permet un libre échange entre verbe et nom peut plus facilement nous surprendre avec des mots comme celui de Bernard Shaw:

(21) A drama critic is a man who leaves no turn unstoned,

qu'une langue qui ne dispose pas de ce moyen. Et ici encore, une traduction univoque, qui ne prendrait pas en considération l'allusion évidente à l'expression sous-jacente (to leave no stone unturned), serait déficiente.

Étant donné ces différences entre les langues, il paraît impossible d'établir des règles ou même des hypothèses universellement valables sur le taux de traduisibilité des jeux de mots. Tout au plus pourrait-on envisager d'établir une sorte d'échelle de traduisibilité pour des couples de langues, ce qui pourrait faire partie d'une analyse contrastive. Et encore faut-il se rendre compte du caractère asymétrique de cette comparaison, car celui qui traduit des jeux de mots français en anglais ne se heurte pas aux mêmes problèmes que celui qui traduit en sens inverse.

Ces problèmes, ne sont-ils pas de nature «civilisationnelle» aussi bien que linguistique ? À première vue, oui, en effet. Mais les problèmes de la première espèce touchent à un problème plus général. C'est que nous pouvons admettre qu'on ne traduit ordinairement que ce qui est intéressant pour un public-cible. Traduire un numéro du Canard enchaîné de 1972 pour un public allemand de 1987 serait vide de sens. Son contenu est si lié à l'actualité temporelle (même pour un Français de 1987 le texte devrait être «traduit»!) aussi bien que géographique, qu'il ne présente plus qu'un intérêt marginal pour les «non-branchés». Digne d'être traduit, normalement parlant, est seulement ce qui a une valeur atemporelle, non actuelle, universelle ou quasi universelle, ou ce qui peut de toute façon s'attendre à un public intéressé. Si l'on regarde la traduction humoristique dans cette perspective, une grande partie des problèmes civilisationnels se trouve être écartée presque automatiquement: il n'est pas trop intéressant de mesurer le taux de traduisibilité de textes que personne ne s'aviserait de traduire... Ainsi il est très peu probable qu'un mot comme (14) puisse jamais se passer d'une explication, s'il devait être offert à un public non francophone. 
Restent donc les obstacles de nature linguistique, Pour en venir à une évaluation de la traduisibilité ou de la non-traduisibilité des ambiguïtés-jeux de mots, on ne peut pas se fonder, malheureusement, sur la façon dont elles ont été effectivement traduites, puisqu'on rencontre couramment des cas de non-traduction ou de traduction inadéquate que l'on pourrait remplacer par des traductions tout à fait satisfaisantes. Ce fait, on l'avait déjà constaté au passage (voir les exemples (9) et (10) cités plus haut). Mais très illustratif aussi, à cet égard, est le résultat d'une comparaison faite de la traduction des jeux de mots d'un album d'Astérix (le Tour de Gaule) en anglais, en allemand et en néerlandais (Van Olphen 1986).

Dans cette étude, Van Olphen a relevé que sur 35 ambiguïtés-jeux de mots en français, la traduction anglaise en avait gardé 34, l'allemande 29 et la néerlandaise 23 . Bien que toutes les traductions données ne soient pas parfaites, ce sont tout de même des pourcentages assez élevés de réussites relatives pour un type de discours réputé «intraduisible», même pour ce qui concerne le néerlandais. À quoi il convient d'ajouter que parmi les 12 cas de non-traduction, il y en a au moins 9 qui auraient très bien pu être restitués convenablement. Contentons-nous de quelques exemples:

(22) [Astérix et Obélix sont sur le point de quitter un Romain attaché dans une chaise de poste; c'est Obélix qui parle le premier et Astérix qui répond]

- Ça m'ennuie de quitter ce Romain. Il est très attachant.

— Laissons-le. Quelqu'un finira par le délivrer.

Dans la traduction néerlandaise, l'isotopie double de attacher d'une part et de délivrer d'autre part se perd complètement:

(22a) - Jammer dat we die Romein achter moeten laten... Hij lijkt me erg aardig!

- Ach ... we komen straks wel meer «aardige» Romeinen tegen!

(= «Dommage de devoir quitter ce Romain. Il a l'air gentil.

Bah! On va rencontrer encore pas mal de Romains "gentils".»)

Néanmoins $I l$ est très attachant aurait pu être traduit par «Hij voelt zich duidelijk met ons verbonden» et la réplique d'Astérix par: «Laat 'm maar. Hij kan zijn post beter niet verlaten». La première traduction rend très bien l'ambiguïté de attachant, la deuxième est assez «libre», il est vrai, mais elle garde la cohérence entre image et texte et maintient l'isotopie double de l'original (post étant à la fois «garde» et «poste»).

(23) [Obélix saute sur un cheval qu'il a déjà monté auparavant. Le cheval s'affaisse sous le poids et, irrité, il pense: - je l'ai toujours sur le dos alors !

Dans la traduction anglaise on rencontre une ambiguïté analogue :

(23a) - Why am I always saddled with him!

tandis que la version néerlandaise, où l'on dispose pourtant de la même solution qu'en anglais (opgezadeld a les mêmes sens propre et figuré), donne simplement:

(23b) - Waarom moet IK hèm toch iedere keer treffen!

(= «Pourquoi toujours tomber sur lui !»)

(24) — Lucius Obtobus, notre village t'offre sa spécialité...

La châtaigne!

Châtaigne est devenu dans la traduction néerlandaise oplawaai (= «coup de poing»), si bien que l'autre sens («marron»), indispensable dans le contexte du banquet, se perd tout à fait. Toutefois la traduction muilpeer (= «coup de poing» avec allusion à poire) ou oorvijg (= «gifle» avec allusion à figue) s'imposerait!

Ce sont autant d'exemples où le traducteur néerlandais n'aurait pas eu besoin de le céder à son collègue anglais pour des raisons linguistiques. Tout ce qu'on peut dire, c'est qu'il s'est visiblement donné moins de peine pour chercher des équivalents que celui-ci, qui s'est montré bien plus inventif. 
Dans un autre mémoire de fin d'études (Oosterwaal 1985), on s'est efforcé de trouver une traduction adéquate, en néerlandais, de 133 jeux de mots français, tirés de diverses sources. Or, il est apparu que pour une trentaine de ces énoncés, une traduction littéralè était disponible ; une cinquantaine de cas pouvaient être traduits avec des expressions ou des notions voisines, susceptibles d'évoquer une image analogue; quarante cas se traduisaient au moyen d'une image différente, il est vrai, mais sans que l'effet pragmatique diffère sensiblement de celui de l'original ; pour treize jeux de mots seulement (c'est-à-dire moins de dix pour cent) il était impossible de trouver une traduction satisfaisante.

Il convient d'ajouter quelques remarques à propos de ces résultats. Normalement une traduction adéquate exige $a$ ) une grande fidélité au texte-source et $b$ ) l'acceptabilité du texte-cible. Cependant, à notre avis cette double exigence doit être légèrement relativisée dans le cas des ambiguités-jeux de mots. Étant donné que la plupart des ambiguïtés voulues se distinguent des énoncés non équivoques par une anomalie rhétorique (cf. Landheer 1984: 47 sq.), la traduction n'a pas besoin non plus d'être conforme à une norme logique stricte, ni (nécessairement) à la norme du «bon usage» conventionnel de la langue-cible, pourvu que la cohérence (macro-)contextuelle soit assurée. Et ensuite, la fidélité au texte-source n'est pas non plus une exigence essentielle dans les cas qui nous occupent: souvent on peut obtenir un effet ambigu analogue, entièrement satisfaisant, en changeant légèrement la forme linguistique et même le contenu du message. Pour atteindre un effet ludique analogue, on peut sans inconvénient sauter d'un type de jeux de mots à un autre : traduire un jeu homonymique par un jeu paronymique, ou inversement, un jeu vertical par un jeu horizontal, et ainsi de suite.

Ainsi le français, ne disposant pas des moyens anglais pour rendre littéralement le mot de Shaw cité plus haut (21), pourrait «se contenter» de :

(21a) Un critique dramatique est quelqu'un grâce a qui toute pièce est taillée en pièces,

tandis que (17) Doux présent du présent peut devenir en néerlandais :

(17a) Schat die 't heden mij schonk,

où le jeu horizontal se maintient sur le plan phonique, tandis qu'il est remplacé sur le plan sémantique par un jeu vertical (schat signifiant «trésor» dans toutes ses acceptions).

Toute langue a ses points forts et ses points faibles qu'il s'agit de connaître et d'exploiter, si l'on veut réexprimer adéquatement une idée originale sous une forme lapidaire.

\section{CONCLUSION}

Lire et traduire des auteurs comme Queneau, Vian, Prévert ${ }^{22}$, Ponge et tant d'autres, veut dire être sensible à leurs virtuosités verbales, être sensible aux surprises de ce que Guiraud (1976: 113) appelle «la défonctionnalisation ludique du langage». Même dans la littérature la plus sérieuse l'isotopie double ou même multiple joue un rôle non négligeable. Notre étonnement devant les cas vraiment très nombreux de non-traduction de telles ambiguittés conscientes a été à l'origine de cet article, qui a d'abord voulu être un essai de démythification du «dogme a priori de l'intraduisibilité» concernant ce type de discours. Sans vouloir nier que sa traduction constitue un défi, demande une extrême habileté, de l'ingéniosité et de la persévérance (sinon un esprit cryptogrammatique !) de la part du traducteur, nous pouvons constater que dans la grande majorité des cas, disons entre 80 et $90 \%$, il est possible de trouver une traduction entièrement satisfaisante. Il faut pour cela exploiter les «points forts» de la langue-cible (qui doit bien être la langue maternelle du traducteur): la polysémie, ou l'homonymie, des structures «polysynthétiques», une grande productivité morphologique, ou bien une grande transparence des 
mots complexes, etc. Chaque phénomène offre des possibilités spécifiques pour manier le discours ludique ou polyvalent. Sur ce point concret, des études contrastives approfondies ne manqueraient pas d'avancer la recherche traductologique. Qu'on aime ou non ce type de discours, cela n'a aucune importance. Négliger ses occurrences sous quelque prétexte que ce soit, mène toujours à une perte sensible dans le texte d'arrivée. L'essentiel de la traduction du jeu de mots n'est ni sa structure linguistique, ni (souvent) le contenu exact de son message, mais plutôt sa valeur pragma-rhétorique. Ce qui importe, c'est moins sa structure interne, sa cohérence micro-contextuelle, que sa structure externe et argumentative, et sa cohérence macro-contextuelle ${ }^{23}$. Le traducteur qui s'évertue à trouver pour telle ou telle ambiguïté-jeu de mots un énoncé synonyme dans la langue-cible n'aboutira pas, bien souvent, mais s'il «s'accommode» de donner un équivalent communicatif, le traducteur bien préparé n'aura pas besoin de décevoir trop souvent son public ${ }^{24}$.

Notes

1. À moins qu'on n'admette avec Guiraud 1976:111, que la surprise (provoquée par un rapprochement inattendu de deux termes) «est un des éléments essentiels du rire».

2. Elles s'opposeraient sous ce rapport aux figures de sens: «À l'encontre des figures de mots, les figures de sens ne dépendent pas du matériel phonique de la langue et sont, du moins en principe, traduisibles». (Ibid. : 42)

3. Le seul «déplacement» qui se produise concerne la perspective métonymique : dans la langue de départ, c'est l'acteur qui est mis en relief, dans la langue d'arrivée, c'est l'action.

4. Pour satisfaire aux exigences de ce type de traduction, il est indispensable, à notre avis, que l'on traduise dans sa langue maternelle, qui, dans notre cas, est le néerlandais. Nous aurons soin cependant d'expliquer les traductions néerlandaises en français.

5. Cf. L. Söll 1968: 166, Hausmann 1974: 107, Guiraud 1976: 102, Newmark 1981 : 108, Colloque «Humour et traduction», Paris, 1985, passim.

6. Ces exemples d'ambiguités lexicales resp. syntaxique ont été empruntés au Dictionnaire de linguistique 1973 : 29 ; cf. aussi le Goffic 1981 : 220 sq. et Landheer $1984: 2$ sq.

7. Ce n'est pas toujours le cas ;] cf. infra l'exemple (14).

8. Cf. Hausmann $1974: 14$ sq., Guiraud $1976: 108$ sq., Landheer $1984: 5-9$.

9. Pour plus de détails, voir Landheer 1987: $105 \mathrm{sq}$

10. Cf. Guiraud $1976: 105$.

11. Nous ne nous arrêterons pas ici sur le problème controversé de la distinction entre polysémie et homonymie, parce que ce problème est sans conséquence pour notre exposé.

12. Jusqu'ici nous n'avons relevé aucun exemple d'un discours ludique fondé sur l'homographie non homophonique.

13. Pour les exemples (12)-(18), nous proposons chaque fois une traduction, ou - dans le cas de (15) - une correction d'une traduction existante.

(12a) Sterven : dichter zijn bij het graf

(= «Mourir : être poète près de la tombe / être plus proche de la tombe»)

14. (13a) Weeg je woorden wel!

(= «Pesez bien vos mots / Ne manquez pas de peser vos mots»)

15. (14a) Reis naar het einde van de nacht ... elijke verveling (combinaison haplologique de «la nuit» et «l'ennui nocturne»)

16. À noter que non seulement (sur les) talons est ambigu ici, mais le nez (sur) aussi. Dans la traduction néerlandaise la première ambiguïté s'est perdue (met zijn neus op de hakken van de twee meisjes), mais elle aurait pu être gardée grâce au mot hielen (au lieu de hakken): op de hielen a un sens propre et un sens figuré qui conviennent parfaitement ici.

17. (16a) Wee het moment waarop je weent

(= «Tu regretteras le moment où tu pleures» + un simulacre de la répétition syntagmatique : wee / weent).

18. Pour la traduction de (17), voir la fin du paragraphe suivant.

19. (18a) Woordenstrijd leidt altijd tot haat en nijd (Ici ce sont la rime et le rythme qui doivent compenser en quelque sorte l'effet homophonique français)

20. Une équipe de l'Université de Leiden a entrepris la traduction de ce recueil de Ponge. Voici la traduction de ce vers pongien :

(18a) Halverwege de kist en het kastje heeft het Nederlands kistje..., in: Erutarettil - Périodique français de l'Université de Leyde, $1(1985,4 / 5)$, p. 17. 
21. Ce qui suit est valable aussi, mutatis mutandis, pour les autres exemples où il n'y a aucun doute sur le statut des deux occurrences.

22. Voir Boyer 1968.

23. La plupart des exemples cités ci-dessus ont été traités comme s'il s'agissait d'énoncés isolés. Nous regrettons en effet de ne pas avoir pu montrer, dans le cadre de cet article, que justement cette cohérence macrocontextuelle jouait un rôle si important dans les traductions proposées (sauf, bien entendu, dans le cas des aphorismes).

24. C'est seulement après avoir terminé la rédaction de cet article que nous avons pu constater que $R$. Zimmer 1981 (surtout 50 sq.) arrive à une conclusion semblable, à propos d'une analyse comparative de cinq traductions différentes de jeux de mots rabelaisiens en allemand.

\section{BIBLIOGRAPHIE}

BALLARD, M. (1987) : «L'ambiguiité», in la Traduction de l'anglais au français, Paris. BERRENDONNER, A. (1981): Eléments de pragmatique linguistique, Paris.

BOYER, R. (1968) : «Mots et jeux de mots», Studia Neophilologica 40, pp. 317-358.

Colloque «Humour et traduction», Paris, décembre 1985.

DEBUSSCHER, G. \& J.P. van NOPPEN (éd.) (1985) : Communiquer et traduire. Hommages à Jean Dierickx. Éd. de l'Université de Bruxelles.

DUBOIS, J. et al. (1973) : Dictionnaire de linguistique, Paris.

ECO, U. (1984) : la Structure absente, Introduction à la recherche sémiotique, Paris.

FRANÇOIS, D. \& F. (1967) : «L'ambiguïte linguistique», Word 23, pp. 150-179.

FUCHS, C. (éd.) (1985): Aspects de l'ambiguité et de la paraphrase dans les langues naturelles, BerneFrancfort-New York.

GUIRAUD, P. (1976) : les Jeux de mots, Paris.

HAUSMANN, F.-J. (1974) : Studien zu einer Linguistik des Wortspiels, Tübingen.

KELLY, L_-G. (1971) : «Punning and the Linguistic Sign», Linguistics 66, pp. 5-11.

KOOH, J.-G. (1971) : Ambiguity in Natural Language, Amsterdam.

LADMIRAL, J.-R. (1979): Traduire : théorèmes pour la traduction, Paris.

LANDHEER, R. (1984) : Aspects linguistiques et pragmatico-rhétoriques de l'ambiguïté, thèse, Leiden.

LANDHEER, R. (1987): «Ambiguilté et paraphrase au niveau traductologique», Colloque international «'Lambiguïté et la paraphrase», Caen, avril.

LE GOFFIC, P. (1981) : Ambiguité linguistique et activité de langage, thèse d'État, Paris.

MOUNIN, G. (1963): les Problèmes théoriques de la traduction, Paris.

NEWMARK, P. (1981): Approaches to Translation, Oxford-New York.

VAN OLPHEN, Ch. (1986): «L'ambiguîté comme problème traductologique - Une analyse comparative», mémoire de fin d'études, Université de Leiden.

OOSTERWAAL, B. (1985): «Aspects traductologiques et pragmatico-ludiques du jeu de mots», mémoire de fin d'études, Université de Leiden.

REBOUL, O. (1984) : la Rhétorique, Paris.

RÉCANATI, F. (1981) : les Énoncés performatifs, Paris.

SELESKOVITCH, D. \& M. LEDERER (1984): Intepréter pour traduire, Paris.

SÖLL, L. (1968) : «Sprachstruktur und Unübersetzbarkeit», Neusprachliche Mitteilungen 21, pp. 161-167.

ZIMMER, R. (1981): Probleme der Ubersetzung formbetonter Sprache, Beih, z. Zschr. f. Rom. Phil. 181, Tübingen. 